\title{
El concepto de debate emergente como recurso para la inclusión de competencias interculturales en la formación inicial docente en Chile ${ }^{1}$
}

\author{
The concept of emerging debate as a resource for the inclusion of \\ intercultural competences in the initial teacher training in Chile
}

\author{
0 conceito 'debate emergente' como recurso para inclusão de \\ competências interculturais na formação inicial de professores no Chile
}

ISSN 1688-9304 - DOI: https://doi.org/10.18861/cied.2019.10.2.2909

Froilán Cubillos Alfaro*2

https://orcid.org/0000-0002-6943-7994

Benjamín Romero Zamora*3

https://orcid.org/0000-0001-7262-3360

Juan José Navarro*4

http://orcid.org/0000-0003-2065-3482

Fecha de recibido: 13/11/2018

Fecha de aprobado: 29/05/2019

\begin{abstract}
Resumen
El presente artículo se orienta a problematizar el desarrollo de herramientas y competencias profesionales orientadas a la interculturalidad en los profesores de Chile, analizando la formación inicial docente en dicho país y su pertinencia para el ejercicio profesional de los profesores en contextos de diversidad cultural con presencia indígena, puntualmente mapuche. Para ello se abordan las condiciones en las que se ha llevado a cabo la escolarización de estudiantes mapuche, además de las características y circunstancias de la implementación hasta la fecha del Programa de Educación Intercultural Bilingüe (PEIB), proyecto que durante las últimas dos décadas se ha constituido en el principal referente en cuanto a la interculturalidad educacional en Chile. Ambos elementos se consideran como antecedentes de referencia para el análisis de la naturaleza y las características de la formación inicial docente que se desarrolla actualmente en las instituciones de educación superior (IES) chilenas, tema tratado en el segundo apartado del artículo. Esto demuestra la tensión existente entre las tendencias del contexto y la práctica universitaria de formación de profesores, ante cuyos resultados se plantea, en un tercer apartado, el concepto de debate emergente como una herramienta epistemológica que permita, facilite y oriente la inclusión transversal de elementos adecuados para una pedagogía intercultural en la trayectoria académica de aquellos estudiantes que serán los futuros docentes chilenos. Palabras clave: educación intercultural, Programa de Educación Intercultural Bilingüe, formación inicial docente, competencias pedagógicas, debates emergentes.
\end{abstract}

\section{Abstract}

The article deals about the development of intercultural tools of teachers in Chile. For such purposes, the article reflects about the preschool teacher training in our country and its pertinence for professional practice in contexts of cultural diversity which includes native population, specifically Mapuche. In this respect, the conditions of Mapuche schooling 
and the characteristics of the Bilingual Intercultural Education Program (PEIB) in Chile are analyzed. Said program has become the main reference in terms of educational interculturality in our country in the two last decades. Both elements are considered as reference antecedents for the analysis of the nature and characteristics of the preschool teacher training that is currently being developed in the higher education institutions, showing the tension between the trends of the contexts and university practices. In view of the above, we propose the concept of emerging debate as an epistemological path that allows the inclusion of appropriate elements for an intercultural pedagogy in the academic trajectory of the future teachers.

Keywords: intercultural education, Bilingual Intercultural Education Program, preschool teacher training, pedagogical competences, emerging debate.

\section{Resumo}

Este artigo visa questionar o desenvolvimento de ferramentas e competências profissionais orientadas para a interculturalidade em professores do Chile, analisando a formação inicial de professores naquele país e sua relevância para a prática profissional deles em contextos de diversidade cultural com presença indígena, pontualmente mapuche. Para tanto, são abordadas as condições em que foi dada a escolarização dos estudantes mapuches, além das características e das circunstâncias da implementação do Programa Intercultural de Educação Bilíngue (PEIB), um projeto que durante as duas últimas décadas se tornou a principal referência em termos de interculturalidade educacional no Chile. Ambos os elementos são considerados referências para a análise da natureza e características da formação inicial de professores atualmente em desenvolvimento nas instituições chilenas de ensino superior (IES). Esse tópico, discutido na segunda seção do artigo, demonstra a tensão existente entre as tendências do contexto e a prática universitária de formação de professores, cujos resultados, em uma terceira seção, suscitam o conceito de um debate emergente como ferramenta epistemológica que permite, facilita e orienta a inclusão transversal de elementos adequados para uma pedagogia intercultural na trajetória acadêmica dos alunos que serão os futuros professores chilenos.

Palabras-chave: Educação intercultural - Programa Intercultural de Educação Bilíngue Formação inicial de professores - Competências pedagógicas - Debate emergente.

\section{Introducción}

El presente artículo busca problematizar la formación inicial docente en Chile desde la perspectiva de la educación intercultural en relación con los pueblos indígenas de este país. Dicho tema se constituye en problemática en el marco de las políticas públicas orientadas a la interculturalidad implementadas durante las últimas décadas (entre las cuales el Programa de Educación Intercultural Bilingüe [PEIB] es el más importante) tendientes a visibilizar, revitalizar y revalorizar la cultura y cosmovisiones de esos pueblos a partir de las demandas y necesidades expresadas por ellos mismos (Ibáñez, Rodríguez y Cisternas, 2015; Turra y Ferrada, 2016). En ese sentido se observa que, si bien se han planteado iniciativas que tienden a la valoración y revitalización de las culturas indígenas de Chile, específicamente en el ámbito educativo, en la práctica estos proyectos han carecido de consistencia tanto estructural como funcional entre sus diversos componentes. Tal es la situación del mencionado PEIB (Lagos, 2015).

En contextos de transformación educativa como lo anteriormente mencionado, el papel que los profesores pueden ejercer en el desarrollo de un proyecto es vital. Sin embargo, en el caso de la educación intercultural, la formación profesional que reciben los estudiantes de pedagogía no ha acompañado los intentos por considerar a las culturas diferentes como valorables y dignas de ser incluidas en la integración del currículum escolar (Arias, 
Quintriqueo y Valdebenito, 2018; Quilaqueo y Quintriqueo, 2017; Turra y Ferrada, 2016). Desarrollamos nuestro análisis a partir de la situación del pueblo mapuche en tanto caso paradigmático. Esto pues, según el Censo 2017, el 12,8\% de la población chilena se considera perteneciente a algún pueblo indígena u originario, universo dentro del cual el 79,8\% se considera mapuche, lo que representa el 9,9\% de la población total del país (INE, 2018, p. 16). De tal manera conforman el grupo mayoritario entre los nueve pueblos originarios reconocidos legalmente en Chile ${ }^{5}$. Esto es, sin negar la situación similar de otros pueblos ni los matices y variaciones que puedan existir entre una realidad y otra.

El artículo se centra, en una primera parte, en el Programa de Educación Intercultural Bilingüe y en las características y circunstancias de su aplicación hasta la fecha, en tanto gran paladín de las políticas orientadas a la interculturalidad en educación en Chile. Un segundo apartado trata acerca de cómo se ha desarrollado en las instituciones de educación superior (IES) chilenas la formación profesional de los profesores, demostrando la tensión existente entre las tendencias del contexto y la práctica universitaria. Finalmente, el último apartado gira en torno al concepto de debates emergentes como una herramienta epistemológica que podría orientar la inclusión transversal del enfoque intercultural en la formación de los docentes en la educación superior.

\section{Escolarización mapuche y el Programa de Educación Intercultural Bilingüe (PEIB) en Chile}

Tradicionalmente, la formación de los niños mapuche se ha realizado al amparo de sus propias comunidades. Sin embargo, desde el siglo XVI, con la llegada de los españoles a los territorios del sur de Chile, se generaron en territorio mapuche dos modelos de formación: el propiamente mapuche y no escolarizado, y el implementado por los conquistadores a través de las empresas misioneras y evangelizadoras, basado en la escritura y lectura del castellano (Turra y Ferrada, 2016). Este paralelismo formativo continuó relativamente, incluso con posterioridad a la independencia de la república, hasta fines del siglo XIX cuando el Estado de Chile llevó a cabo la ocupación de la Araucanía. Este hecho marca hasta hoy la relación entre el Estado chileno y el pueblo mapuche, para quien significó la pérdida de su autonomía político-cultural y el comienzo de un proceso de pauperización y forzada aculturación (Turra y Ferrada, 2016).

Según Lagos (2015), debido a esto los indígenas comenzaron a recibir protección legal desde la década de 1930 a través de una serie de decretos y leyes de diversa índole en favor de sus comunidades. Sin embargo, las políticas públicas en torno al mundo indígena no han superado la dimensión asistencialista, cuantificadora y asimilacionista, lo que ha sido funcional para reproducir hasta el día de hoy la asimetría económica, social, cultural y política en las relaciones entre la sociedad nacional y el mundo indígena (Lagos, 2015, p. 85).

En el ámbito educativo, la institución escolar se ha desarrollado como civilizadora y de carácter unitario y homogeneizante. Por ende, históricamente la escolarización de niños indígenas se ha realizado sobre la base de finalidades y contenidos educativos monoculturales occidentales que se contraponen al conocimiento indígena (Morales, Quintriqueo, Uribe y Arias, 2018). Ante esto, inicialmente el sistema formativo mapuche se replegó a una esfera privada, al espacio familiar inmediato, configurándose como una instancia de oposición y/o resistencia al modelo educativo oficial representado por la escuela occidental (Turra y Ferrada, 2016; Quilaqueo y Quintriqueo, 2017). Sin embargo, a gran escala y a lo largo del tiempo, para ellos, evitar la escolarización no pudo ser una estrategia a largo plazo. De hecho, se observa un alejamiento de los niños mapuche de la educación tradicional de su pueblo pues, a pesar de todo, la escuela es percibida por la 
población mapuche como una oportunidad de movilidad social y económica. Este proceso de escolarización ha traído consecuencias relacionadas con el desarraigo de los niños con respecto a sus familias y comunidades, con la pérdida progresiva de la autoidentificación de las nuevas generaciones y con la pérdida parcial o total de la lengua mapuche en tanto elemento vital para la construcción de la identidad mapuche (Quilaqueo y Quintriqueo, 2017).

Según su propia percepción, la experiencia escolar de las poblaciones indígenas en el territorio mapuche se caracterizó por constituir una experiencia de discriminación, marginalidad y castigo por ser ellos fenotípica (apariencia), lingüística y culturalmente diferentes (Fuenzalida y Casas, 2010, p. 77). En el mismo sentido, una investigación de Carbonell muestra que el contacto de los niños indígenas con la escuela les ha generado un trauma en tres dimensiones: psicológica, lingüística y cultural. “En el aspecto psicológico, enfrentan estereotipos de inferioridad, basados en el desprecio de los profesores hacia su lengua materna, hábitos y valores diferentes, que generan en el niño temor e inseguridad. En el aspecto lingüístico, al ingresar a la edad escolar el niño ya ha estructurado su lengua, que en general es negada junto con su cultura, deseando liberarse de la estigmatización que significa. En el aspecto cultural, constituye un contenido central de la socialización, que al ser negado en la educación escolar afecta en una pérdida progresiva de su identidad sociocultural. El conjunto de dichos factores son de vital importancia para el desarrollo de una educación intercultural en el contexto mapuche" (Carbonell citado en Morales et al., 2018, p. 57).

Posteriormente, a partir de la década de los noventa el Estado asumió un rol más protagónico en la definición de una política pública global orientada hacia el mundo indígena dado el convencimiento de la existencia de una deuda que debía ser saldada. Esto explica la identificación de los indígenas como destinatarios específicos de políticas sociales vinculadas a tierras, créditos y educación (Abarca y Zapata, 2007). En este marco, uno de los pilares fundamentales fue la consideración de la educación y sus lenguas originarias en tanto ejes centrales de la revitalización de las culturas indígenas como parte del nuevo trato que el Estado quería entablar con estas poblaciones, pues se entienden ambos ámbitos como primordiales para concretar el reconocimiento, respeto y protección de sus culturas (Lagos, 2015). A este respecto también hay opiniones que proponen que la promoción de esta política estatal se debió más a demandas externas que poco tenían que ver con los pueblos indígenas en sí o con el interés estatal por su desarrollo, pues haberse negado no solo hubiese significado el repudio de países observantes de los derechos humanos y de organizaciones como Unesco o Unicef, sino que podría haber frenado las inversiones extranjeras en nuestro país en caso de generarse un conflicto abierto debido a ello (Quintrileo, Yáñez y Valenzuela, 2013).

Igualmente, en el marco de este proceso se promulgó en 1993 la Ley 19.253, Ilamada Ley Indígena. A través de esta ley el Estado chileno reconoció oficialmente la existencia de nueve pueblos originarios en su territorio: Mapuche, Aimara, Rapa Nui o Pascuenses, Atacameños, Quechuas, Collas y Diaguitas en el norte del país; y Kawashkar o Alacalufe y Yámana o Yagán en los canales australes (Ley 19.253, art. 1). En su artículo 32 esta Ley ordena que la Corporación Nacional de Desarrollo Indígena (CONADI) en conjunto con el Ministerio de Educación (MINEDUC) desarrolle un modelo de educación que atienda, en sus especificidades culturales, a los niños de las poblaciones y comunidades de alta concentración indígena (Fuenzalida y Casas, 2010; Quintrileo et al., 2013). Posteriormente, como producto de esta política, el Ministerio de Educación implementó en 1996 el Programa de Educación Intercultural Bilingüe (PEIB), cuyo objetivo principal era "contribuir a mejorar 
los logros de aprendizaje, a partir del fortalecimiento de la identidad étnica de las niñas y los niños de establecimientos educacionales de Educación Básica ubicados en contextos de diversidad cultural y lingüística"6. Dicho programa centra su acción en torno a tres ejes: contextualización curricular, participación comunitaria y enseñanza de las lenguas indígenas (MINEDUC, 2017).

Siguiendo a Walsh (2010), la interculturalidad se puede desglosar en dos dimensiones: la interculturalidad relacional, que pone énfasis en el contacto entre culturas, ya sea en condiciones igualitarias o desiguales, y la interculturalidad funcional, que se centra en el reconocimiento de la diversidad dentro de estructuras sociales complejas. Asimismo plantea la interculturalidad crítica en tanto objetivo que debe buscarse a través de la voluntad de diversidad de actores sociales.

El Programa de Educación Intercultural Bilingüe (PEIB) nace, entonces, para instalar una educación compensatoria para indígenas, tendiente a mejorar su rendimiento y sus posibilidades de ascenso social.

Esto se fundamenta en la existencia de un bajo rendimiento en los estudiantes indígenas debido a los siguientes factores: (a) la descontextualización del currículo en relación con la cultura local; (b) el desarrollo de prácticas pedagógicas inadecuadas para los intereses, necesidades y aprendizajes de estos; y (c) la exclusión de las lenguas vernáculas (Lagos, 2015 , p. 86). Por ello, este programa ha propuesto, en el discurso, un enfoque pedagógico que se plantea de la siguiente manera: (a) de revalorización y rescate de las culturas indígenas dentro de una lógica de una sociedad pluralista que aspira a la búsqueda de un diálogo entre culturas; (b) como un nuevo método de educación intercultural para la revitalización de los pueblos indígenas comenzando por su lengua, que es a la vez, por excelencia, el símbolo de su cultura; y (c) como una propuesta pedagógica que pretende difundir las costumbres y culturas indígenas en el ámbito escolar a través del aprendizaje de su lengua, promoviendo el conocimiento y respeto por la diversidad (Lagos, 2015, p. 87). Sin embargo, a dos décadas de la implementación de dicho programa se concluye que el Programa de Educación Intercultural Bilingüe se ha constituido en una política pública que reproduce las relaciones asimétricas y las representaciones sociales sobre el sujeto indígena que propone superar: el indígena subordinado al "civilizado", sin voz ni agenda propia, en condiciones precarias, con bajo estatus y cuyos sistemas locales de producción/ transmisión de conocimiento carecen de legitimidad. Así, la dimensión indígena queda reducida al plano del folclore y sin mayor pertinencia cultural ni complejización respecto de lo que es ser indígena hoy (Lagos, 2015, p. 87).

En el análisis que hace Lagos (2015) del PEIB queda expuesto que el programa está comprometido con la interculturalidad solo a medias, y que el producto generado no se corresponde con los objetivos planteados ya que: a) no es una herramienta de revitalización de las lenguas indígenas; b) no cumple con su promesa de ser bilingüe; y c) no es intercultural pues no hay un diálogo simétrico entre las dos culturas en contacto (Lagos, 2015). De hecho, desde el punto de vista de la competencia comunicativa pueden señalarse serias limitaciones e inconsistencias en este programa, cuyo compromiso declarado es la revitalización de las lenguas indígenas en el contexto local y nacional (Quintrileo et al., 2013, p. 59). Según el análisis de estos últimos autores, la propuesta muestra una escasa preocupación por la enseñanza de la lengua, restándole valor como medio de transmisión cultural o dimensión relevante e indisociable de la cultura (Quintrileo et al., 2013, p. 58). Esto podría explicarse porque en este modelo de educación intercultural predomina el componente relacional puesto que hay una operacionalización de la interculturalidad que no cuestiona las estructuras institucionales y sociales en que se pueden llevar a cabo las 
relaciones entre culturas (Quintrileo et al., 2013, p. 52).

Siguiendo a estos autores, la propuesta del PEIB promueve una identidad indígena estática y estereotipada, que ignora el dinamismo de las identidades sociales, culturales y étnicas, así como la evolución de los contextos, donde la lengua es instrumento de comunicación, de interacción y de transmisión unívoca de la cultura (Quintrileo et al., 2013, p. 59).

En virtud de lo anterior, el Programa de Educación Intercultural Bilingüe, en particular, y las políticas estatales sobre educación intercultural en general, exigen ser repensados y reformulados en función de los nuevos contextos indígenas, urbanos y rurales (Lagos, 2015). En esta reformulación el papel que se les conciba y atribuya a los docentes debe ser fundamental.

\section{Interculturalidad en la formación inicial docente}

Según la Unesco (2009) el mayor avance en el pensamiento educativo internacional del último tiempo ha sido el reconocimiento de las múltiples interpretaciones de la realidad y las diversas formas de enseñar y aprender (citado en Hinojosa y López, 2016, p. 90). Ante esto, el enfoque educativo intercultural puede definirse como una aproximación teórica, práctica y metodológica que permite estudiar y comprender las formas de relacionarse entre sujetos que pertenecen a culturas y sociedades diversas (Arias et al., 2018; Morales et al., 2018) fundamentada en dos dimensiones: 1) una relación simétrica donde se genera un diálogo de poder entre los grupos y colectividades socioculturales para la toma de decisiones y de reconocimiento recíproco, y 2) reconocimiento de los respectivos marcos epistémicos de los saberes y conocimientos educativos. Estos son aspectos centrales para desarrollar una educación intercultural, valorando las diferencias sociales y culturales (Morales et al., 2018). Estas relaciones horizontales y mutuamente enriquecedoras es lo que De Sousa Santos denomina diálogo de saberes (De Sousa Santos, 2009; 2010).

A nivel general, el desarrollo de un proyecto de educación intercultural no se puede lograr sin educadores que cuenten con una adecuada formación en competencias interculturales. De hecho, el avance teórico y normativo de la educación en y para la diversidad cultural ha subrayado la necesidad de adecuar la formación inicial del profesorado al carácter plural de la realidad social y educativa (Hinojosa y López, 2016, p. 89). Así, queda demostrado el papel central que desempeña la calidad de las instituciones formadoras de profesores (San Martín, Villalobos, Muñoz y Wyman, 2017). Por lo tanto, de la mano de proyectos como el PEIB, las universidades -en su rol social y producto de su papel en el desarrollo e implementación de tendencias y políticas educativas- deben reflexionar acerca de la concepción, del enfoque y del alcance que se da a la dimensión intercultural en la formación inicial de sus estudiantes de Pedagogía.

Sin embargo, las definiciones de política pública en este ámbito no se han traducido en transformaciones en el currículum de formación de profesores que recojan las especificidades culturales y laborales aplicables en los contextos de indigeneidad (Hinojosa y López, 2016; Turra y Ferrada, 2016) o al menos solo gradualmente, lo que puede ser debido al alto grado de autonomía, a la orientación al mercado y al clivaje organizacional de las propias universidades (San Martín et al., 2017). En el caso de las universidades pertenecientes al Consejo de Rectores de Chile (CRUCH), hasta el año 2013 solo había tres casos en los que se expresaba algún tipo de orientación pedagógica hacia la educación inclusiva, pero esto solo estaba visibilizado en asignaturas específicas (San Martín et al., 2017), de manera esporádica y poco consistente (Hinojosa y López, 2016). 
Considerando lo anterior, tratar el tema de la formación inicial docente desde el enfoque intercultural requiere tener en cuenta tanto las características de la formación profesional como la manera en que esta formación se reflejará en la práctica profesional de los futuros profesores.

En relación a lo primero, el foco se instala en el proceso formativo al que se enfrentan los aspirantes a docentes, problematizando la centralidad del currículo de los programas y los distintos enfoques e instancias para generar procesos inclusivos (San Martín et al., 2017). Los programas eficaces en la inserción de los docentes en aulas altamente diversas se han destacado especialmente por tres aspectos: a) la existencia de planes de estudio que enfatizan la importancia del cómo enseñar y la importancia del contexto en la práctica docente; b) el énfasis en la inserción temprana de los estudiantes -a través de la práctica profesional- que incentive el trabajo colaborativo entre docentes novatos y expertos; y c) la generación de trabajos reflexivos sobre la experiencia en aula (San Martín et al., 2017). Sin embargo, algunos de los problemas identificados en la formación profesional de docentes son los siguientes: (a) omisión de otras corrientes curriculares; (b) nula descripción de supuestos teóricos que están a la base; y (c) omisión de la participación del profesorado y miembros del sistema educativo local y nacional (Arias et al., 2018). Se observa que persiste una formación inicial docente basada en la monoculturalidad occidental, lo que trae como consecuencia el desconocimiento del contexto social y cultural de los estudiantes, tanto en los formadores de profesores como en los futuros profesores (Arias et al., 2018).

En efecto, la mayoría de los programas y el currículum de educación superior para profesores incluye herramientas a nivel de metodologías y didácticas que se centran en el aprendizaje de un alumno promedio, sin considerar la diversidad del estudiantado (Arias et al., 2018; San Martín et al., 2017). Por ello, los egresados de Pedagogía en Chile no están preparados para enfrentar la diversidad que existe en el aula y asimismo poseen un aprendizaje comparativamente menor al de otros países, debilidad reconocida por los propios docentes (San Martín et al., 2017). Los profesores reciben una formación eurocéntrica monocultural que es a su vez transversal a todo el sistema educativo chileno, lo que se refleja en la descontextualización de los contenidos disciplinares que enseñan en las escuelas, influenciados por un discurso social estereotipado, prejuicioso y racista hacia todo lo relacionado con lo mapuche, reproduciendo el colonialismo mediante la educación escolar (Arias et al., 2018; Quilaqueo y Quintriqueo, 2017; Turra y Ferrada, 2016). Esta omisión de conocimientos propios en los actuales programas de formación inicial se traduce en la invisibilización de los niños indígenas y la consecuente desigualdad en la educación de esos estudiantes (Morales et al., 2018). Ante esto se hace necesario formar al profesorado en competencias interculturales orientadas a desarrollar prácticas pedagógicas contextualizadas. Al hablar de prácticas pedagógicas se incluyen formas de hablar, actuar, trabajar, disciplinar, representar el conocimiento y mostrar el saber, a partir de lo cual los profesores integran recursos pedagógicos diversos de manera selectiva (Quilaqueo y Quintriqueo, 2017, p. 123).

Estas competencias interculturales están asociadas a: (a) la necesidad de llevar a cabo la formación inicial docente desde un enfoque educativo intercultural, teniendo en cuenta la realidad social y cultural del país; y (b) la necesidad de que la formación inicial docente contribuya al desarrollo de habilidades específicas relacionadas a la incorporación de las características socioculturales de la población estudiantil (Arias et al., 2018, p. 11).

Estas competencias profesionales deben fundarse sobre cinco ejes: 1) el eje de la contextualización de su práctica pedagógica; 2 ) el eje de los recursos humanos y educativos a disposición; 3) el eje epistemológico, asumiendo la coexistencia de saberes interactuantes 
en las salas de clases; 4) eje de temporalidad, referido a la interacción profesor-estudiante en la progresión de los estudiantes; y 5) el eje de la reflexibilidad y autocrítica acerca de la [propia] labor docente (Arias et al., 2018, p. 14). Por lo tanto, en la medida en que el futuro profesor desarrolle competencias profesionales interculturales podrá ajustar críticamente sus prácticas pedagógicas al contexto social y cultural en el cual se desenvuelva.

En definitiva, para avanzar en el desarrollo de prácticas pedagógicas más contextualizadas, es necesario que se consideren los significados que se construyen en la interacción social entre formador de formadores, futuros profesores, escuela, familia y comunidad puesto que son estos actores sociales quienes están implicados en los procesos educativos escolares (Arias et al., 2018, p. 14).

Ahora, para analizar cómo este tipo de formación profesional experimentada por los futuros docentes influye en su desenvolvimiento profesional hay que aclarar que las escuelas constituyen un espacio donde los estudiantes de ascendencia mapuche ponen en juego los saberes propios y aquellos adquiridos en su formación escolar, en un ambiente de doble racionalidad (Quilaqueo y Quintriqueo, 2017). Esto supondría la capacidad de los docentes de generar en forma permanente una relación entre el saber disciplinar y el conocimiento propio de la profesión docente como un saber pedagógico para la formación intercultural (Quilaqueo y Quintriqueo, 2017), o un diálogo entre el saber occidental y el propio de las comunidades (De Sousa Santos, 2009; 2010).

En caso de no existir esta capacidad en los docentes, la coexistencia de lógicas de conocimiento mapuche y occidental en el medio escolar genera un problema de relaciones sociales en el plano de las prácticas pedagógicas, en la definición de contenidos del currículum escolar y en el significado mismo de la educación escolar (Quilaqueo y Quintriqueo, 2017), lo que se constituye en un problema cotidiano y de no fácil solución para los docentes que lo enfrentan. Asimismo, la distancia entre el conocimiento familiary el conocimiento escolar en el contexto mapuche genera un obstáculo para el aprendizaje significativo, lo que se asocia también a problemas de interacción y relación social entre estudiantes mapuche, no mapuche y los propios profesores. Para comprender esta distancia epistemológica los temas centrales deberían ser los tipos de conocimientos, los criterios de verdad y las fuentes del saber y conocimiento (Quilaqueo y Quintriqueo, 2017).

Por tanto, es necesario conocer cómo los niños indígenas han construido sus conocimientos educativos, desde la base de una educación familiar, en una relación dinámica entre sus comunidades (Quilaqueo y Quintriqueo, 2017, p. 149). Para ello es fundamental formar a los profesionales de la educación en un estilo pedagógico para responder profesionalmente desde la comprensión y el respeto a la diversidad a partir del principio transversal de contextualización sociocultural de la información (Morales et al., 2018).

Sin embargo, la invisibilización de los conocimientos educativos propios de los pueblos indígenas desde los itinerarios formativos de los programas de formación inicial docente incide en la monoculturalidad de las prácticas pedagógicas de los futuros profesores, producto del desconocimiento del contexto sociocultural de los estudiantes (Arias et al., 2018, p. 5).

Esta monoculturalidad se caracteriza por: 1) la práctica monolingüe basada en la lengua oficial del grupo social dominante que niega la incorporación de otras o que minimiza su participación; 2) prácticas que excluyen a las minorías en el desarrollo de las clases; y 3) el uso [irrestricto] del currículum de corte eurocéntrico occidental, el cual desarrolla los conocimientos y valores que la sociedad occidental considera válidos, invisibilizando los saberes, conocimientos y valores que poseen los estudiantes de diferentes culturas (Arias et al., 2018, p. 11). Dicha lógica de pensamiento subyace en el sistema escolar chileno como un 
legado colonial que es transmitido a los estudiantes mediante el currículum escolar, el que se constituye en un dispositivo que perpetúa la monoculturalidad (Arias et al., 2018, p. 3). Finalmente, la distancia entre la lógica del conocimiento escolar y del indígena que la educación debería salvar es considerada, por el contrario, como un aspecto relacionado con la institucionalización monocultural del conocimiento científico occidental y la desvaloración del saber indígena, cuya racionalidad es ignorada al definir contenidos y finalidades educativas escolares (Quilaqueo y Quintriqueo, 2017). Así, la educación escolar chilena es en gran parte la responsable de las representaciones simbólicas que transmiten patrones que coinciden con la cultura de los grupos dominantes de la sociedad, imponiendo cíclicamente la hegemonía del conocimiento occidental en la escuela y la sociedad (Arias et al., 2018, p. 10). Esta condición es un elemento fundamental en el proceso de epistemicidio, esto es, la destrucción de conocimientos propios de los pueblos causada por el colonialismo europeo (De Sousa Santos, 2009; 2010).

El resultado es, por ende, que los estudiantes del sistema educativo, ya sean niños mapuche, no mapuche o inmigrantes, adquieren las normas de comportamiento, valores y conocimientos de la sociedad dominante, omitiendo sus particularidades, reproduciéndose de esta forma el racismo institucional (Arias et al., 2018).

En el contexto descrito anteriormente queda claro que las universidades, como espacio de formación de formadores, tienen mucho que decir.

\section{La interculturalidad como debate emergente en la formación inicial docente}

De hecho, durante su última convocatoria, la Conferencia Regional de Educación Superior (CRES, 2018) reconoció la existencia de una deuda de los estados y de las sociedades latinoamericanas, en general, y de las universidades y otras instituciones de educación superior en particular con respecto a los pueblos indígenas y a los afrodescendientes. Ante esto expresa la obligación ética, social, jurídica y política que las universidades y otros tipos de IES tienen para asegurar la aplicación de sus derechos, así como la de educar contra el racismo y todos sus modos de discriminación (CRES, 2018 p. 14).

En ese escenario, y considerando todo lo expuesto anteriormente, se hace necesaria una constante reflexión sobre el quehacer de las instituciones formadoras de profesores. En este marco la educación del profesorado en el ámbito intercultural en el contexto nacional requiere de una sólida formación investigativa y vocacional (Morales et al., 2018).

En el entendido de que, en teoría, los procesos de formación docente deben caracterizarse por atender y asumir en los trayectos de formación diversas demandas sociales -entre ellas la integración-, el currículum permanentemente se condiciona para contener, a lo menos, una visión general de los sujetos a quienes está dedicado (Geeregat, Vásquez y Fierro, 2012). Pero, por el contrario, a la formación docente se le critica por no capacitar adecuadamente para la práctica docente pues está desprovista de conocimientos pedagógicos y sobrecargada de saberes, tratándose más bien de una formación academicista (Morales et al., 2018) y poco contextualizada.

Entonces, hay que dar espacio a la idea de que el discurso de la academia también tiene un impacto considerable en las definiciones sobre la relación entre currículum y sociedad, y a partir de ello los modos en los que se reconfiguran las nociones de currículum en la universidad (Morelli, 2017, p. 69). Es por ello que el currículum universitario cuenta con el desafío de elaborar discursos complejos configurados por diversidad de saberes, reconociendo que la formación universitaria está plagada de contingencias (Morelli, 2017, p. 74). Estas contingencias pueden encontrarse en la tensión entre lo local y lo global, donde debe definirse el currículum. 
Esta tensión cobra una dimensión específica en el caso del campo curricular, ya que hace referencia a las decisiones globales consideradas por las políticas educativas y universitarias y a las definiciones locales que pueden encontrarse en las unidades académicas como pequeños escenarios configurados por el contexto, la práctica y los sujetos. El reconocimiento de esta tensión en los estudios del currículum es clave porque construye discurso de la emergencia como circunstancia (Morelli, 2017).

En este contexto, una forma a través de la cual se puede desarmar el esquema cíclico monocultural de la formación docente y su posterior expresión en el ámbito escolar es como un debate emergente, entendido por Morelli (2017) como "aquellas problemáticas, discusiones y demandas culturales compartidas por grupos sociales que, instalados como denuncia contingente, merecen ser considerados en la formación universitaria" (p. 71). Como ejemplos de debates emergentes pueden mencionarse la inclusión social, la multiculturalidad o interculturalidad, la educación ambiental, las alfabetizaciones múltiples, los estudios de género y el lugar de lo femenino (Morelli, 2017, p. 75), entre otros.

Los debates emergentes se ubican entre los problemas sociales y el saber científico, y surgen subjetivamente del antagonismo social al impedir al sujeto ser él mismo, tendiendo a la exclusión de las identidades sociales (Geeregat et al., 2012; Morelli, 2017). La decisión de considerar a las problemáticas sociales actuales en el currículum universitario deja lugar a la denuncia de lo que queda escindido y negado como problemática social y, para el caso de la educación superior, tratadas por fuera de los espacios curriculares, nunca como parte de éstos (Morelli, 2017). En el caso de la formación docente para la interculturalidad se busca que el proceso formativo se desarrolle en un marco de límites más flexibles, que admitan el tratamiento de contenidos disciplinares con los propios del medio socio-familiar. Un aspecto de relevancia para contextualizar el currículo es el descubrir e investigar en el propio medio cuáles son los contenidos que dan sentido y significado al proceso pedagógico (Morales et al., 2018).

La adopción de este enfoque intercultural incorpora perspectivas más reflexivas y complejas que ayuden a los futuros docentes a valorar críticamente la incidencia de las condiciones sociales, económicas y políticas en la construcción de la identidad y el desarrollo personal y grupal (Hinojosa y López, 2016, p. 90). De lo contrario, el currículum universitario de la formación docente queda como un objeto estático, técnicamente condicionado para cumplir su compromiso político y social (Morelli, 2017). Cuando se logra, la formación docente para la diversidad cultural tiene un impacto importante en la modificación de las concepciones previas y las creencias, genera una comprensión más profunda de la cultura, significados más amplios de la enseñanza en contextos multiculturales y una concienciación crítica incipiente (Hinojosa y López, 2016, p. 94). Las propuestas formativas con más impacto han sido las que se preocupan por vincular teoría y práctica e incorporar la reflexión crítica, el diálogo, el intercambio con compañeros, los estudios de caso y el trabajo colaborativo (Hinojosa y López, 2016, p. 102).

Esta perspectiva comprensiva y dialógica del currículum abandona la antigua concepción del conocimiento único y apuesta por la necesidad de interrogarlo y comprenderlo desde su contexto (Quilaqueo y Quintriqueo, 2017, p. 132), o instalarse desde el punto de vista que autores como Walsh (2010) denominan la de-colonialidad, que se sustenta en una praxis que construya una conciencia de subjetividad reflexiva que incluya a educandos y educadores y que se constituye en un medio para visibilizar y desestructurar un modelo educativo en el que subyace un orden y una lógica todavía sustentados en estructuras sociales, políticas y epistémicas coloniales (citado en Geeregat et al., 2012). 
La introducción de las demandas mapuche por una formación docente pertinente a su contexto sociocultural puede entenderse desde este punto de vista como un elemento a considerar en los debates emergentes en el ámbito universitario. Así, se produciría una renegociación del saber indígena ante su marginalización, una oportunidad para considerarlo en los contextos educativos locales, utilizando una epistemología pertinente que recoja las formas de producción del conocimiento que no están sustentadas en la racionalidad científica occidental (Quilaqueo y Quintriqueo, 2017, p. 135; De Sousa Santos, 2010).

Como hemos visto, sin embargo, el conocimiento indígena, mapuche para nuestro caso, ha sido ignorado e invisibilizado, a modo de círculo vicioso, entre el currículum escolar y el currículum universitario en la formación docente. Por lo anterior, nuestra causa por una educación intercultural debe entenderse de manera crítica respecto del rol transformador que debe tener la formación de los profesores en relación a los fundamentos que sostienen la asimetría y desigualdad en la sociedad chilena mediante la escolarización (Quilaqueo y Quintriqueo, 2017). Este planteamiento puede considerarse un paso adelante en la vía que circulan las instituciones de educación superior en la actualidad, y particularmente en Chile, hacia una formación profesional pertinente y adecuada a las demandas del medio, así como al establecimiento de una relación más cercana y dinámica entre las instituciones de educación superior y la sociedad a la que sirven.

\section{Conclusiones}

Las demandas mapuche por una educación pertinente a su cultura como una forma de reconocimiento a sus particularidades socioculturales pueden datarse desde el primer tercio del siglo XX. Debido a ello, desde la última década del siglo pasado el Estado chileno reconoció esa necesidad y ha venido implementando una serie de políticas públicas educativas que buscan superar esta situación, entre las cuales el Programa de Educación Intercultural Bilingüe puede considerarse la más importante. Sin embargo, a dos décadas de su instauración los resultados de estas iniciativas están lejos de lo esperado, entre otras cosas por su inconsistencia estructural y funcional, por un financiamiento inadecuado y por la descoordinación de las distintas instancias que en ellas intervienen. En el contexto de estas tendencias la formación inicial docente no ha acompañado el itinerario del resto de las políticas en este sentido.

De hecho, aun en la actualidad se evidencia una tendencia general a la monoculturalidad de las prácticas pedagógicas en las carreras de Pedagogía, lo que se refleja a su vez en una monoculturalidad en las prácticas de esos futuros profesores en su desempeño profesional, traduciéndose en la descontextualización de las experiencias escolares de niños mapuche, en el incumplimiento de la palabra de las políticas públicas respecto al rescate y revitalización de la cultura de los pueblos indígenas y en la disfuncionalidad de la labor docente en muchos contextos.

Así, la escolarización monocultural se constituye en un importante factor de construcción social de las relaciones entre lo mapuche y no mapuche y cumple un rol colonizador e integrador de las nuevas generaciones de niños mapuche a la matriz social y cultural eurocéntrica instalada en el país (Quilaqueo y Quintriqueo, 2017) ya que no cuestiona las estructuras de dicha matriz.

Ante esta situación proponemos el acercamiento de estas demandas a las instituciones de educación superior en calidad de debates emergentes (Morelli, 2017) como una manera de integrar dentro de las instancias de formación profesional de los profesores las demandas 
sociales, en este caso interculturales desde círculos mapuche, que puedan influir en su trayectoria académica flexibilizando las estructuras estudiantiles, ampliando la base epistemológica sobre la que trabajan los profesores (De Sousa Santos, 2009) e incentivando el desarrollo de su espíritu crítico y reflexivo. El objetivo es mejorar la formación inicial docente, con ello mejorar sus prácticas pedagógicas y en último término mejorar la calidad del aprendizaje escolar de todos los estudiantes de nuestro país en un contexto que se autodefine como amplio, pluralista y democrático.

\section{Referencias bibliográficas}

Abarca, G. y Zapata, C. (2007). Indígenas y educación superior en Chile: el caso mapuche. Revista Calidad en la Educación (26), 57-79.

Arias, K., Quintriqueo, S. y Valdebenito, V. (2018). Monoculturalidad en las prácticas pedagógicas en la formación inicial docente en La Araucanía, Chile. Educ. Pesqui, São Paulo, 44. Recuperado de: http://www.scielo.br/pdf/ep/v44/1517-9702-ep-S1678-4634201711164545. pdf

Conferencia Regional de Educación Superior, CRES (2018). Educación superior, diversidad cultural e interculturalidad en América Latina. Colección CRES 2018, coordinado por Daniel Mato. Córdoba: UNESCO, IESALC y UNC. Recuperado de: http://bibliotecadigital.cin.edu. ar/bitstream/123456789/2277/1/Educaci\%C3\%B3n\%20superior\%2c\%20diversidad\%20 cultural.pdf

De Sousa Santos, B. (2009). Una epistemología del Sur. México: Consejo Latinoamericano de Ciencias Sociales (CLACSO)/Siglo XXI.

De Sousa Santos, B. (2010). Descolonizar el saber, reinventar el poder. Uruguay: Ediciones Trilce. Recuperado de: http://www.boaventuradesousasantos.pt/media/Descolonizar\%20 el\%20saber final\%20-\%20C\%C3\%B3pia.pdf

Fuenzalida, P. y Casas, M. (2010). La educación intercultural bilingüe como campo de tensión política entre el mundo mapuche - williche y el estado. Revista Líder, 16(12), 73-84. Recuperado de: https://go.galegroup.com/ps/anonymous?id=GALE\%7CA353323252\&sid= googleScholar\&v=2.1\&it=r\&linkaccess=abs\&issn=07170165\&p=IFME\&sw=w

Geeregat, O., Vásquez, O. y Fierro, J. (2012). Procesos de formación inicial docente en contextos multiculturales: inclusión y exclusión. Estudios pedagógicos (Valdivia), 38(1), 345 351. Recuperado de: https://dx.doi.org/10.4067/S0718-07052012000100021

Hinojosa Pareja, E. y López, M.C. (2016). Impacto de la formación inicial docente intercultural. Una revisión de la investigación. Convergencia, 23(71), 89-109.

Ibáñez, N., Rodríguez, M.S. y Cisternas, T. (2015). La educación intercultural desde la perspectiva de docentes, educadores tradicionales y apoderados mapuche de la región de la Araucanía: Una co-construcción. Fondo de Investigación y Desarrollo en Educación (FONIDE). Recuperado de: http://peib.mineduc.cl/wp-content/uploads/2018/05/F811345 INFORME_FINAL_IBANEZ_UMCE.pdf 
El concepto de debate emergente como recurso para la inclusión de competencias interculturales...

INE (2018). Síntesis de Resultados Censo 2017. Santiago: Instituto Nacional de Estadísticas. Recuperado de: https://www.censo2017.cl/descargas/home/sintesis-de-resultadoscenso2017.pdf

Lagos, C. (2015). El Programa de Educación Intercultural Bilingüe y sus resultados: ¿perpetuando la discriminación? Pensamiento Educativo. Revista de Investigación Educacional Latinoamericana, 52(1), 84-94. Recuperado de: http://pensamientoeducativo.uc.cl/files/ journals/2/articles/676/public/676-2138-1-PB.pdf

MIDEPLAN (1993). Ley 19.253. Establece normas sobre protección, fomento y desarrollo de los indígenas, y crea la Corporación Nacional de Desarrollo Indígena. Recuperado de: https://www.leychile.cl/Navegar?idNorma=30620

MINEDUC (2017). Programa de Educación Intercultural Bilingüe 2010-2016. Santiago de Chile: Ministerio de Educación. Recuperado de: http://peib.mineduc.cl/wp-content/ uploads/2018/05/20180226-PEIB-2010-2016-Versi\%C3\%B3n-Final.pdf

Morales, S., Quintriqueo, S., Uribe, P. y Arias, K. (2018). Interculturalidad en educación superior: experiencias en educación inicial en la Araucanía, Chile. Convergencia, 25(77), 5576. Recuperado de: http://www.redalyc.org/jatsRepo/105/10555363003/html/index.html

Morelli, S. (2017). El currículum universitario y la relación con el saber. Nociones desde la posmodernidad. Investigación cualitativa, 2(2), 68-82. Recuperado de: https://ojs. revistainvestigacioncualitativa.com/index.php/ric/article/view/63/44

Quilaqueo, D. y Quintriqueo, S. (2017). Métodos educativos mapuche: retos de la doble racionalidad educativa. Aportes para un enfoque educativo intercultural. Temuco: Universidad Católica de Temuco.

Quintrileo, C., Yáñez, C. y Valenzuela, C. (2013). Una aproximación crítica a la propuesta en consulta del Programa de Educación Intercultural Bilingüe (PEIB) en Chile. Logos: Revista de Lingüística, Filosofía y Literatura, 23(1), 45-61.

San Martín, C., Villalobos, C., Muñoz, C. y Wyman, I. (2017). Formación inicial docente para la Educación Inclusiva. Análisis de tres programas chilenos de pedagogía en Educación Básica que incorporan la perspectiva de la educación inclusiva. Revista Calidad en la educación, (46), 20-52. Recuperado de: https://dx.doi.org/10.4067/S0718-45652017000100020

Turra, O. y Ferrada, D. (2016). Formación del profesorado en la lengua y cultura indígena: una histórica demanda educativa en contexto Mapuche. Educ. Pesqui., São Paulo, 42(1), 229- 242.

Walsh, C. (2010). Interculturalidad crítica y educación intercultural. En J. Viaña, L. Tapia y C. Walsh (Eds.), Construyendo interculturalidad crítica. Instituto Internacional de Integración Andrés Bello: Bolivia, 75-96. 


\section{Notas}

* Universidad Metropolitana de Ciencias de la Educación (Chile).

${ }^{1}$ Este artículo fue realizado en el marco del Proyecto de Investigación “Sistematización del currículum crítico emergente de la escuela Kom Pu Lof Ñi Kimeltuwe para la elaboración de una propuesta de innovación didáctica y evaluativa" (PMI-EXA/PNII/02/2017).

2 Doctor en Didáctica de las Ciencias Sociales, Universidad Autónoma de Barcelona (España). Magister en Didáctica de las Ciencias Sociales, Universidad de Alicante (España). Geógrafo, Universidad de Chile. Director, Departamento de Historia y Geografía, Universidad Metropolitana de Ciencias de la Educación (Chile).

${ }^{3}$ Magíster en Educación, mención Pedagogía y Gestión Universitaria, Universidad Metropolitana de Ciencias de la Educación (Chile). Licenciado en Historia y Profesor de Historia y Geografía, Universidad de Concepción (Chile).

${ }^{4}$ Licenciado en Educación con mención en Geografía y Profesor de Historia, Geografía y Educación Cívica, Universidad Metropolitana de Ciencias de la Educación (Chile).

${ }^{5}$ La Ley 19.253 de 1993, en su artículo 1, reconoce oficialmente a los pueblos Mapuche (79,8\%), Aimara (7,2\%), Rapa Nui o Pascuenses (0,4\%), Atacameños (1,4\%), Quechuas (1,5\%), Collas (0,9\%), Diaguitas $(4,1 \%)$ Kawashkar o Alacalufe $(0,1 \%)$ y Yámana o Yagán $(0,1 \%)$. Entre paréntesis el \% respectivo en relación al total de la población que se identifica con alguno de ellos, según datos del censo 2017.

${ }^{6}$ Véase http://www.sipi.siteal.iipe.unesco.org/politicas/622/programa-de-educacion-interculturalbilingue-peib.

\section{Contribución de autoría:}

La concepción del trabajo científico pertenece al Dr. Froilán Cubillos Alfaro y al Mg. Benjamín Romero. La recolección, interpretación y análisis de datos estuvo a cargo del Mg. Benjamín Romero. La redacción/ revisión del manuscrito fue realizada por el Dr. Froilán Cubillos Alfaro, el Mg. Benjamín Romero y el Lic. Juan José Navarro. Todos los autores revisaron y aprobaron el contenido final. 